\title{
Oxidation of Electron-Deficient Phenols Mediated by Hypervalent Iodine(V) Reagents: Fundamental Mechanistic Features Revealed by a DFT-Based Investigation
}

\author{
Mona Jalali, ${ }^{\mathrm{a}}$ Alex C. Bissember, ${ }^{\mathrm{a}}$ Brian F. Yates, ${ }^{\mathrm{a}}$ Sarah E. Wengryniuk, ${ }^{* \mathrm{~b}}$ and Alireza \\ Ariafard, $*$ a \\ a School of Natural Sciences - Chemistry, University of Tasmania, Hobart, Tasmania 7001, \\ Australia \\ ${ }^{\mathrm{b}}$ Department of Chemistry, Temple University, Philadelphia 19122, USA
}

\begin{abstract}
Hypervalent iodine(V) (HVI) compounds are highly efficient reagents for the double oxidative dearomatization of electron-rich phenols to o-quinones. We recently reported that an underexplored class of iodine $(\mathrm{V})$ reagents possessing bidentate bipyridine ligands, termed $\operatorname{Bi}(N)$-HVIs, could efficiently dearomatize electron-poor phenols for the first time. To better understand the fundamental mechanistic basis of this unique reactivity, density functional theory (DFT) was utilized. In this way, different pathways were explored to determine why $\mathrm{Bi}(N)$-HVIs are capable of facilitating these challenging transformations while more traditional hypervalent species, such as IBX cannot. Our calculations reveal that the first redox process is the rate-determining step, the barrier of which hinges on the identity of the ligands bound to the iodine(V) center. This crucial process is composed of three steps: (a) ligand exchange, (b) hypervalent twist, and (c) reductive elimination. We found that strong coordinating ligands disfavour these elementary steps and, for this reason, HVIs bearing such ligands cannot oxidize the electron-poor phenols. In contrast, the weakly coordinating triflate ligands in $\mathrm{Bi}(N)$-HVIs allow for the kinetically favorable oxidation of such phenols $\left\{\right.$ e.g., $\Delta \mathrm{G}^{\ddagger}=\sim 22 \mathrm{kcal} / \mathrm{mol}$ where $\operatorname{Bi}(N)=\operatorname{Bi}\left(4-C_{2}\right.$ Etbipy $\left.)\right\}$. It was also identified that trapping triflic acid, which is generated in situ, is a key role played by the basic bidentate bipyridine ligands in $\mathrm{Bi}(N)$-HVIs as this serves to minimize decomposition of the sensitive ortho-quinone product.
\end{abstract}




\section{Keywords}

Density functional theory (DFT); phenol oxidation, bidentate nitrogen ligand, hypervalent iodine reagents, ortho-quinone, reaction mechanism

\section{INTRODUCTION}

Hypervalent iodine (HVI) compounds represent a class of important and versatile reagents and reactants in organic synthesis. ${ }^{1}$ For example, iodine $(\mathrm{V})$ species, such as 2-iodoxybenzoic acid (IBX), are recognized as effective oxidants for a wide range of synthetic transformations. ${ }^{2}$ More specifically, the double oxidation of phenols by IBX or related iodine(V) reagents delivers ortho-quinones in a highly regioselective manner. ${ }^{3}$ However, to date, a notable limitation of iodine(V)-mediated phenol oxidation chemistry is that these transformations are typically restricted to electron-rich phenol substrates.

Bidentate nitrogen-ligated iodine(V) compounds, $\mathrm{Bi}(N)$-HVIs, were first reported by Zhdankin and coworkers in $2002 .{ }^{4}$ Recently, we demonstrated that these compounds, and specifically $\mathrm{Bi}\left(4-\mathrm{CO}_{2}\right.$ Etbipy)-HVI, were uniquely effective at facilitating the highly efficient double oxidative dearomatization of electron-deficient phenols, such as $p$-nitrophenol (Table 1, entries 1 and 2). ${ }^{5}$ In this report, the reactivity of $\mathrm{Bi}\left(4-\mathrm{CO}_{2}\right.$ Etbipy)-HVI was benchmarked against a range of other iodine(V) reagents. Specifically, IBX provided very low yields of the target quinone and precursor $\mathrm{PhI}(\mathrm{O})(\mathrm{OAc})_{2}$ gave no reaction at all (entries 3 and 4). The stronger oxidant $\mathrm{PhI}(\mathrm{O})(\mathrm{OTf})_{2}$ afforded the product quinone in $59 \%$ yield after 20 minutes, which decreased to $27 \%$ yield after 4 hours due to continued oxidative degradation (entries 5 and 6 ). Interestingly, $\mathrm{Bi}(4-O M e$ Etbipy)-HVI provided the product in much lower yields in comparison to $\mathrm{Bi}\left(4-\mathrm{CO}_{2}\right.$ Etbipy)-HVI (entry 7). This revealed that electronic effects within the bipyridine ligand might play a crucial role in the efficiency of the oxidation. It was also interesting to note that the reaction employing pyridine-ligated $\mathrm{PhI}(\mathrm{O})(\mathrm{Py})_{2}(\mathrm{OTf})_{2}$ only returned unreacted 
starting material, indicating a bidentate ligand may also be required for desired dearomatization.

Table 1. Reported dearomatization of electron-deficient phenols to ortho-quinones by various iodine $(\mathrm{V})$ reagents: selected results. ${ }^{5}$

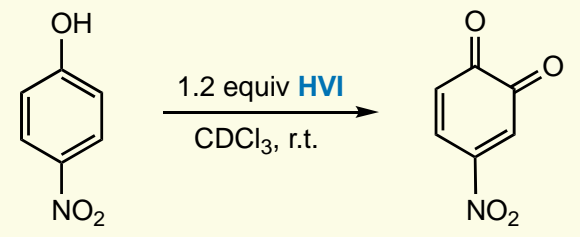

\begin{tabular}{cccc}
\hline entry & HVI oxidant & time $(\mathbf{h})$ & yield (\%) \\
$\mathbf{1}$ & $\mathrm{Bi}\left(4-\mathrm{CO}_{2} \mathrm{Etbipy}\right) \mathrm{HVI}$ & 0.5 & 99 \\
$\mathbf{2}$ & $\mathrm{Bi}\left(4-\mathrm{CO}_{2} \mathrm{Etbipy}\right) \mathrm{HVI}$ & 4 & 91 \\
$\mathbf{3}$ & $\mathrm{IBX}$ & 24 & 12 \\
$\mathbf{4}$ & $\mathrm{Phl}(\mathrm{O})(\mathrm{OAc})_{2}$ & 24 & 0 \\
$\mathbf{5}$ & $\mathrm{Phl}(\mathrm{O})(\mathrm{OTf})_{2}$ & 0.66 & 59 \\
$\mathbf{6}$ & $\mathrm{Phl}(\mathrm{O})(\mathrm{OTf})_{2}$ & 4 & 27 \\
$\mathbf{7}$ & $\mathrm{Bi}(4-\mathrm{OMebipy}) \mathrm{HVI}$ & 0.66 & 38 \\
$\mathbf{8}$ & $\mathrm{Phl}(\mathrm{O})(\mathrm{Py})_{2}(\mathrm{OTf})_{2}$ & 0.66 & 0 \\
\hline
\end{tabular}

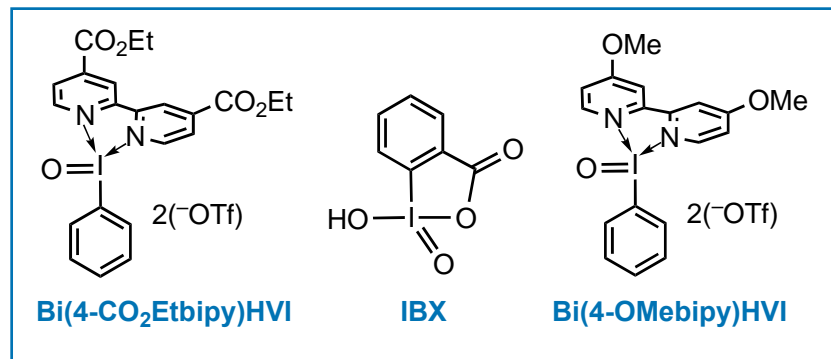

The above-mentioned experimental results prompted us to comprehensively investigate the mechanism of the oxidation of $p$-nitrophenol by the above-mentioned iodine(V) oxidants with the aim of answering the following questions: (i) Why are $\mathrm{Bi}\left(4-\mathrm{CO}_{2}\right.$ Etbipy)-HVI and $\mathrm{PhI}(\mathrm{O})(\mathrm{OTf})_{2}$ potent oxidants in this chemistry, while IBX and $\mathrm{PhI}(\mathrm{O})(\mathrm{OAc})_{2}$ are not? (ii) Is there a difference between the reactivity of $\mathrm{Bi}\left(4-\mathrm{CO}_{2}\right.$ Etbipy)-HVI compared to $\mathrm{PhI}(\mathrm{O})(\mathrm{OTf})_{2}$ ? (iii) If not, why is the reaction efficiency for the former greater than that for the latter? (iv) Why does the replacement of $\mathrm{Bi}\left(4-\mathrm{CO}_{2}\right.$ Etbipy)-HVI with $\mathrm{Bi}$ (4-OMebipy)-HVI considerably 
decrease the product yield? Why does the use of pyridine rather than a bipyridine attenuate the reactivity of the iodine(V) reagent? We anticipated that employing Density Functional Theory (DFT) to answer the foregoing questions would provide an enhanced understanding of the fundamental processes involved in iodine(V)-mediated oxidations. Furthermore, by revealing how the structure of $\mathrm{Bi}\left(4-\mathrm{CO}_{2}\right.$ Etbipy)-HVI specifically relates to its function, we thought that our study could serve as a platform from which the design of new hypervalent iodine(V) reagents and associated organic transformations would be better enabled.

\section{RESULTS AND DISCUSSION}

We commenced our DFT investigation by evaluating the stability of different isomers of $\mathrm{Bi}(4-$ $\mathrm{CO}_{2}$ Etbipy)-HVI. The four lower energy isomers of $\mathrm{Bi}\left(4-\mathrm{CO}_{2}\right.$ Etbipy)-HVI are shown in Figure 1a. This found that structure 1 , with a distorted octahedral geometry, is the most stable of these. In this complex, the oxo, bipyridine ligand, and a triflate anion occupy equatorial positions, while the second triflate occupies the axial position trans to the phenyl ligand. It is worth noting that due to the strong trans influence of the phenyl ligand, the iodine center binds more weakly to the axial triflate than to the equatorial triflate. This is reflected in the longer $\mathrm{I}-\mathrm{O}^{b}$ bond distance within 1. Similarly, the strong trans influence of the oxo ligand results in unsymmetrical coordination of bipyridine, which is consistent with the longer distance between the I and $\mathrm{N}^{\mathrm{b}}$ atoms in comparison to the I and $\mathrm{N}^{\mathrm{a}}$ atoms. Other isomers are less stable than $\mathbf{1}$ by $1.6 \mathrm{kcal} / \mathrm{mol}$ (isomer 2), $4.7 \mathrm{kcal} / \mathrm{mol}$ (isomer 3), and $10.1 \mathrm{kcal} / \mathrm{mol}$ (isomer 4). The lower stability of five-coordinate ion pair $\mathbf{4}$ compared to other isomers indicates that the triflate ligand trans to the phenyl is more likely to remain bound to the iodine center (although weakly). We also found that the most stable structure $\mathbf{1}$ is resistant to complete dissociation of the bipyridine and triflate ligands. This is supported by the predicted endergonicity of these processes (Figure 1b). The same is true for other isomers (see Figures S1 and S2). 
A recent study investigating the stability of isomers of a related $\mathrm{Bi}$ (bipy)-HVI species also found that an isomer structure analogous to structure $\mathbf{1}$ is the most stable. ${ }^{6}$ This consistency suggests that regardless of the electronic nature of the bidentate nitrogen ligand, isomer $\mathbf{1}$ is likely lower in energy than the other possible systems.

(a)

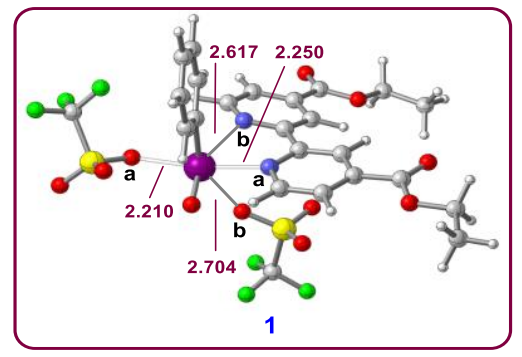

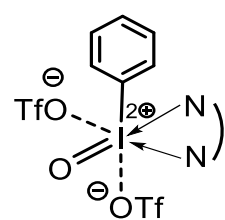

1

$0.0(0.0)$

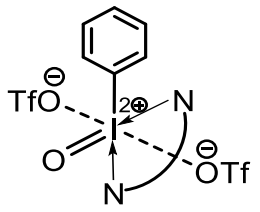

3

$4.7(2.4)$

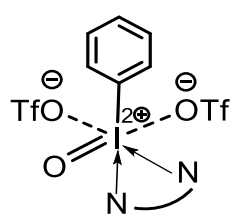

2

$1.6(2.4)$<smiles>O=P(O)(O)c1ccccc1</smiles>

4

(b)

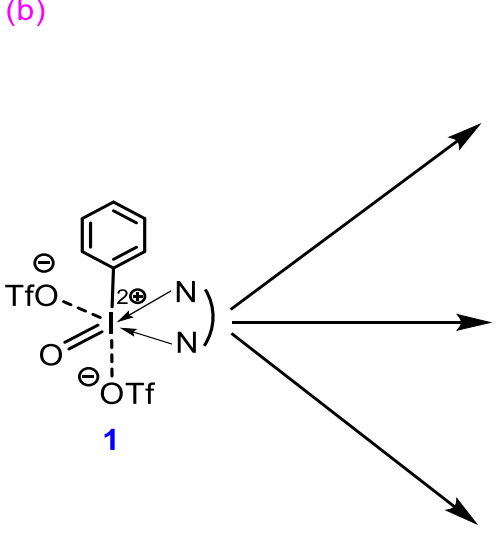

1

TfO

5<smiles>c1ccccc1</smiles>
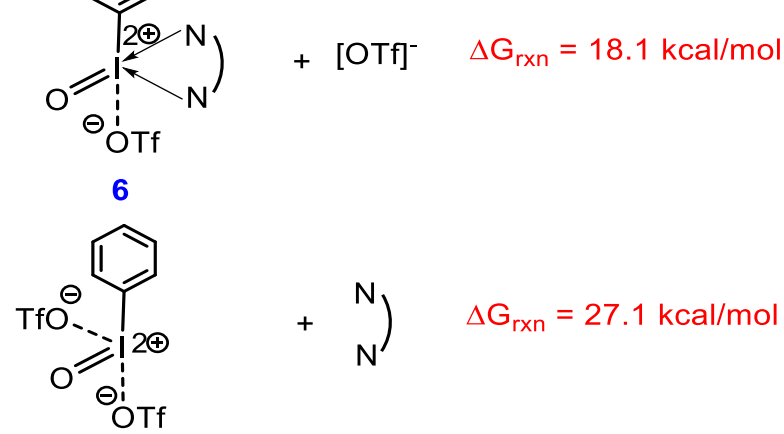

$\left.+\begin{array}{l}\mathrm{N} \\ \mathrm{N}\end{array}\right) \quad \Delta \mathrm{G}_{\mathrm{rxn}}=27.1 \mathrm{kcal} / \mathrm{mol}$

6'

Figure 1. (a) Stability of different isomers of $\mathrm{Bi}\left(4-\mathrm{CO}_{2}\right.$ Etbipy)-HVI. (b) Reaction free energies for dissociation of the triflate and bipyridine ligands from 1. Free energies calculated by SMD/M06-2X /def2-TZVP//SMD/M06-2X/LANL2DZ(d),6-31G(d) in chloroform are given in kcal/mol.

Oxidation of $\boldsymbol{p}$-nitrophenol by Bi(bipy)-HVI. Next, we focused on understanding why $\mathrm{Bi}(4-$ 
$\mathrm{CO}_{2}$ Etbipy)-HVI is a suitable reagent for oxidation of electron-deficient phenols in contrast to IBX (Table 1, entries 1 and 3). ${ }^{5}$ To this end, different mechanistic pathways were explored for the oxidation of $\mathrm{PhOH}$ by $\mathrm{Bi}\left(4-\mathrm{CO}_{2}\right.$ Etbipy $)-\mathrm{HVI}$, the results of which are given in the Supporting Information (see Figures S3 and S4). Scheme 1 outlines the most favorable pathway for the phenol oxidation identified from our DFT studies. This reaction involves the following key steps: (i) ligand exchange between phenol and $\mathrm{Bi}\left(4-\mathrm{CO}_{2}\right.$ Etbipy)-HVI, giving adduct 7 stabilized by in-situ-generated triflic acid; (ii) formation of complex $\mathbf{8}$ via addition of triflic acid to the bipyridine ligand; (iii) generation of intermediate 10, obtained by the dissociation of 9 from $\mathbf{8}$, followed by an isomerization; (iv) the first redox process; (v) tautomerization assisted by the triflate ligand via sequence $11 \rightarrow 12 \rightarrow 13$; and, finally, (vi) the second redox process that forms ortho-quinone 15.

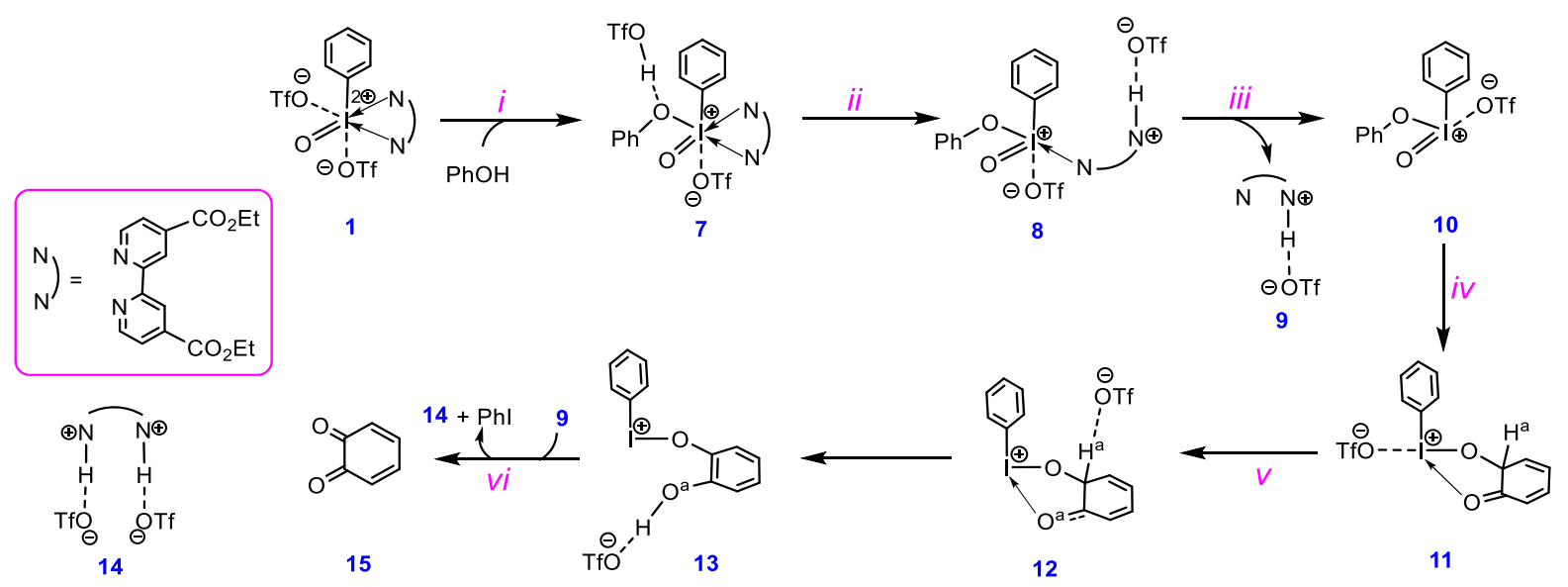

Scheme 1. Key steps for phenol oxidation mediated by 1 derived from our DFT investigation.

Figure 2 shows the energy profile calculated for oxidative dearomatization of $p$-nitrophenol as a model for an electron-deficient substrate based on the mechanism outlined in Scheme 1. Accordingly, the reaction commences by formation of hydrogen-bonded adduct $\mathbf{1 6}$, followed by a ligand exchange involving the concerted interchange associative (CIA) mechanism ${ }^{7}$ through transition structure $\mathbf{T S}_{\mathbf{1 6}-\mathbf{1 7}}$ to provide adduct $\mathbf{1 7}$ which is stabilized by a hydrogen bonding interaction between in-situ-generated triflic acid and the phenolate ligand. This step is 
calculated to occur with an activation barrier of around $11 \mathrm{kcal} / \mathrm{mol}$ and is endergonic by about $8 \mathrm{kcal} / \mathrm{mol}$. Following this, the resulting triflic acid protonates the bipyridine nitrogen trans to the oxo ligand $\left(\mathrm{N}^{b}\right)$ in a thermodynamically favorable process to give intermediate 19 . Due to the $\pi$ conjugation within the bipyridine ligand, the addition of the proton to $\mathrm{N}^{\mathrm{b}}$ significantly reduces the basicity of $\mathrm{N}^{\mathrm{a}}$ and, thus, it binds less strongly to the iodine(V) center in $\mathbf{1 9}$. This is consistent with the longer $\mathrm{I}-\mathrm{N}^{\mathrm{a}}$ distance in $\mathbf{1 9}(2.426 \AA)$ than in $\mathbf{1 8}(2.356 \AA)$ (Figure 2). In other words, $\mathrm{N}^{\mathrm{b}}$ protonation reduces the coordinative stability of the iodine(V) molecule and it changes from a rather inert to a more labile complex. ${ }^{8}$ The dissociation of 9 from $\mathbf{1 9}$ produces 20 in a thermoneutral fashion which then isomerizes to more stable structure 21_OTf. Since the phenolate provides a weaker trans influence relative to the phenyl, the triflate binds more strongly to the iodine atom in 21_OTf, resulting in this complex being more stable than 20. This is consistent with the shorter I-OTf bond distance within 21_OTf $(2.252 \AA)$ in comparison to the equivalent bond in $\mathbf{2 0}(2.412 \AA)$ (Figure 2). It is established that a hypervalent twist must take place for the iodine oxidant to be sufficiently reactive toward the redox process. ${ }^{9,10}$ The corresponding twist occurs by overcoming an activation barrier of $8.4 \mathrm{kcal} / \mathrm{mol}$ via TS $_{21-22}$ OTf which forms 22_OTf. The approach of the ortho-carbon of the phenolate to the oxo ligand in 22_OTf promotes the first redox process via an associative mechanism to afford 23_OTf. ${ }^{10 a}$ The formation of this species (23_OTf) is calculated to be exergonic as much as $36.3 \mathrm{kcal} / \mathrm{mol}$, suggesting that the first redox process is not reversible.

The second redox process commences with formation of outer-sphere complex $\mathbf{2 4}$ formed by dissociation of the triflate ligand from 23_OTf. Due to the weakly coordinating nature of the triflate anion, the outer-sphere complex 24 is only $9.1 \mathrm{kcal} / \mathrm{mol}$ higher in energy than the innersphere complex 23_OTf. The triflate anion within $\mathbf{2 4}$ is then predicted to act as a base and through a deprotonation reaction via $\mathbf{T S}_{\mathbf{2 4 - 2 5}}$ produces Zwitterion-type complex $\mathbf{2 5}$ with an overall activation barrier of $16.2 \mathrm{kcal} / \mathrm{mol}$. The delivery of the resultant triflic acid to the 
anionic oxygen of the catecholate ligand within $\mathbf{2 5}$ gives $\mathbf{2 6}$ (a tautomer of 24) from which the second redox process proceeds via transition structure $\mathbf{T S}$ 26-27. The deprotonation of the hydroxy group in $\mathbf{2 6}$ by the triflate anion increases the electron density on the catecholate ligand, resulting in two electrons from it being transferred to I(III) center via the curly arrow mechanism (Figure 2). Notably, due to the weakly coordinating nature of the triflate anion, inner-sphere complex 28 is only $4.0 \mathrm{kcal} / \mathrm{mol}$ lower in energy than the outer-sphere complex 26. As such, according to our calculations, the overall activation barrier for the second redox step (via $\mathbf{T S}_{\mathbf{2 6 - 2 7}}$ ) is only $12.3 \mathrm{kcal} / \mathrm{mol}$. The triflic acid generated in the last step then reacts with 9 and, consistent with the experimental observations, ${ }^{5}$ gives diammonium salt 14 in an exergonic fashion. From the foregoing computational data, we conclude that the ratedetermining step of this transformation is the first redox step via sequence 21_OTf $\rightarrow$ 22_OTf $\rightarrow$ 23_OTf with an activation barrier of $20.8 \mathrm{kcal} / \mathrm{mol}$. This relatively low free energy of activation is in agreement with the experimental observations and explains why the dearomatization reaction by $\mathrm{Bi}\left(4-\mathrm{CO}_{2}\right.$ Etbipy)-HVI occurs at room temperature (Table 1). 

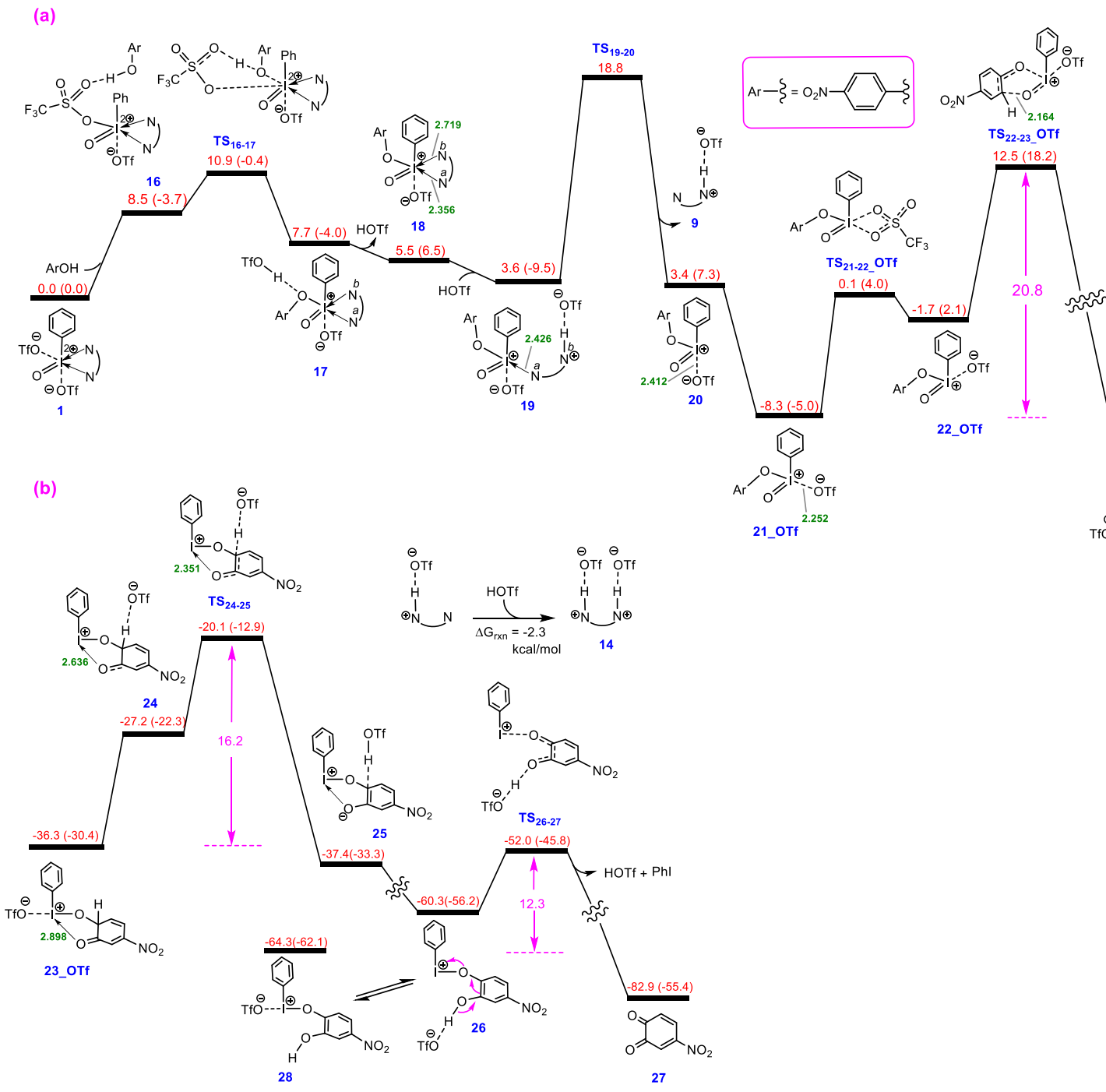

Figure 2. Calculated energy profile for oxidation of $p$-nitrophenol by $\mathrm{Bi}\left(4-\mathrm{CO}_{2}\right.$ Etbipy)-HVI. Free energies (potential energies) calculated by SMD/M06-2X/def2-TZVP//SMD/M062X/LANL2DZ(d),6-31G(d) in chloroform are given in $\mathrm{kcal} / \mathrm{mol}$ and selected bond distances (green values) in $\AA$.

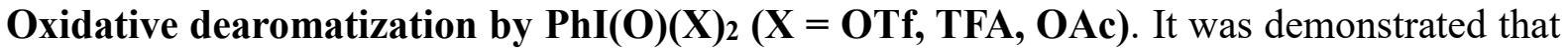
$\mathrm{PhI}(\mathrm{O})(\mathrm{OTf})_{2}$ can mediate the oxidation of $p$-nitrophenol, while $\mathrm{PhI}(\mathrm{O})(\mathrm{OAc})_{2}$ gives no reaction (Table 1, entries 4 and 5). ${ }^{5}$ Furthermore, it was noted that the ortho-quinone product formed by reaction with $\mathrm{PhI}(\mathrm{O})(\mathrm{OTf})_{2}$ decomposed as the reaction time increased (Table 1, entries 6). ${ }^{5}$ This observation suggests that the presence of the Lewis basic bipyridine ligand in $\mathrm{Bi}\left(4-\mathrm{CO}_{2}\right.$ Etbipy)-HVI plays an important role in reducing product degradation. Thus, we employed DFT to investigate and understand the underlying reasons for the reactivity differences between $\mathrm{PhI}(\mathrm{O})(\mathrm{OTf})_{2}$ and $\mathrm{PhI}(\mathrm{O})(\mathrm{OAc})_{2}$. For benchmarking purposes, we also 
studied the oxidation of $p$-nitrophenol by $\mathrm{PhI}(\mathrm{O})(\mathrm{TFA})_{2}$ computationally to predict the reactivity of this oxidant. As discussed in the previous section, the first redox process is the rate-determining step of the oxidative dearomatization. Consequently, for these three systems we limited our calculations to studying this part of the reaction mechanism. As shown in Figure 3, the dearomatization commences with the ligand exchange between $\operatorname{ArOH}$ and $\operatorname{PhI}(\mathrm{O})(\mathrm{X})_{2}$ $\left(\mathrm{X}=\right.$ OTf, TFA, OAc) via the CIA mechanism, ${ }^{7}$ followed by a hypervalent twist and then the redox process via the nucleophilic attack of the ortho-carbon of the phenolate to the oxo ligand. The computed energy profiles given in Figure 3, revealed several noteworthy points.

(i) The coordinating ability of the $\mathrm{X}$ ligand decreases in the following order: $\mathrm{X}=\mathrm{OAc}>\mathrm{TFA}$ > OTf, supported by the shortest I-X bond distance in 29_OAc (2.101 $\AA$ ), and the longest one in 29_OTf $(2.141 \AA)$.

(ii) The overall activation barrier for the oxidative dearomatization increases in the order $\mathrm{X}=$ OTf $<$ TFA $<$ OAc. This suggests that the identity of the X-ligand is an important factor in determining the ease of the redox process. Specifically, $\mathrm{PhI}(\mathrm{O})(\mathrm{OAc})_{2}$ bearing strongly coordinating acetate ligands has a barrier of $34.5 \mathrm{kcal} / \mathrm{mol}$, while $\mathrm{PhI}(\mathrm{O})(\mathrm{OTf})_{2}$, with weakly bound triflate ligands has a computed activation barrier of only $21.7 \mathrm{kcal} / \mathrm{mol}$. This finding is in agreement with the experimental observations and clearly explains why $\mathrm{PhI}(\mathrm{O})(\mathrm{OTf})_{2}$ is an appropriate oxidant for the dearomatization reaction, while $\mathrm{PhI}(\mathrm{O})(\mathrm{OAc})_{2}$ gives no reaction.

(iii) The activation barrier for the ligand exchange via $\mathbf{T S 3 0 - 3 1 \_ X}$ is mainly reliant on the nature of the X-ligand and increases along with the coordinating ability of the X-ligand. This is because it involves a late transition structure, evident from the notable elongation of the $\mathrm{I}-\mathrm{X}$ bond from 29_X to TS30-31_X (Figure 3). The stronger the I-X bond, the more destabilized the

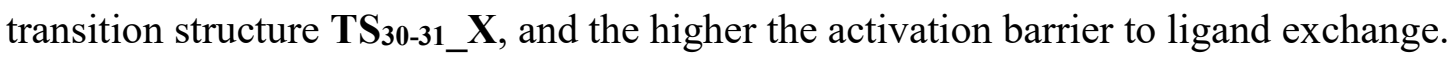

(iv) The ligand exchange represents an almost thermoneutral process, regardless of the nature of the $\mathrm{X}$-ligand, which is consistent with the small values calculated for $\Delta \mathrm{G}_{1}$ listed in Table 2 . 
(v) The hypervalent twist process is more energetic for a stronger coordinating X-ligand. This is supported by the largest value of $\Delta \mathrm{G}_{2}$ for $\mathrm{X}=\mathrm{OAc}$ and the smallest one for $\mathrm{X}=\mathrm{OTf}$ (Table 2). This result can be explained by the low tendency of a relatively strong coordinating Xligand to occupy a position trans to the strong $\sigma$-donor oxo ligand within 22 _X.

(vi) The energy barrier of the redox step (transformation $\mathbf{2 2} \mathbf{X} \rightarrow \mathbf{T} \mathbf{S}_{22-23}$ X) is particularly sensitive to the nature of the X-ligand. As the coordinating ability of the X-ligand increases, the activation barrier to this step increases (see the $\Delta \mathrm{G}^{\ddagger}$ 1 values in Table 2). Consequently, a strong coordinating X-ligand alleviates the electron deficiency of the iodine(V) center in 22_X, making it less susceptible to be involved in the redox step. This hypothesis finds support from the inspection of the NPA charge on the iodine atom in 22_X, which increases along the series from +2.544 in 22_OAc to +2.568 in 22_OTf (Figure 3). Also, this feature causes the redox step for a relatively strong coordinating X-ligand to take place via a later transition structure (TS22-23_X), consistent with the shortest $\mathrm{C}^{\cdots} \mathrm{O}$ O distance in TS22-23_OAc $(2.125 \AA)$ and the longest one in TS22-23_OTf (2.164 A, Figure 3).

(vii) The overall activation barrier to the first redox process is determined by the following formula: $\Delta \mathrm{G}_{\mathrm{t}}^{\dagger}=\Delta \mathrm{G}_{1}+\Delta \mathrm{G}_{2}+\Delta \mathrm{G}^{\dagger}$ (Table 2). As discussed above, $\Delta \mathrm{G}_{1}$ is mainly independent from the nature of the $\mathrm{X}$-ligand and thus only $\Delta \mathrm{G}_{2}$ and $\Delta \mathrm{G}^{\ddagger}$ contribute to the ease of the dearomatization process. A strong coordinating X-ligand makes both transformations 21_X $\rightarrow$ 22_X (hypervalent twist) and 22_X $\rightarrow$ TS22-23_X (redox step) unfavorable, and as a result such a ligand is not appropriate for the phenol oxidation.

(viii) The first redox process by $\mathrm{PhI}(\mathrm{O})(\mathrm{OTf})_{2}$ proceeds with an overall activation energy of $21.7 \mathrm{kcal} / \mathrm{mol}$ (Figure 3a), which is comparable to that of $20.8 \mathrm{kcal} / \mathrm{mol}$ where the process is driven by $\mathrm{Bi}\left(4-\mathrm{CO}_{2}\right.$ Etbipy)-HVI (Figure 2a). This result implies that these two reagents should have a very similar reactivity and the presence of the bipyridine ligand in the latter does not attenuate the oxidizing capacity of the iodine $(\mathrm{V})$ center. This suggests that the presence of the 
bipyridine ligand allows for trapping of the in-situ-generated triflic acid, which considerably reduces the rate of decomposition of the sensitive ortho-quinone product, as shown in Table 1. 


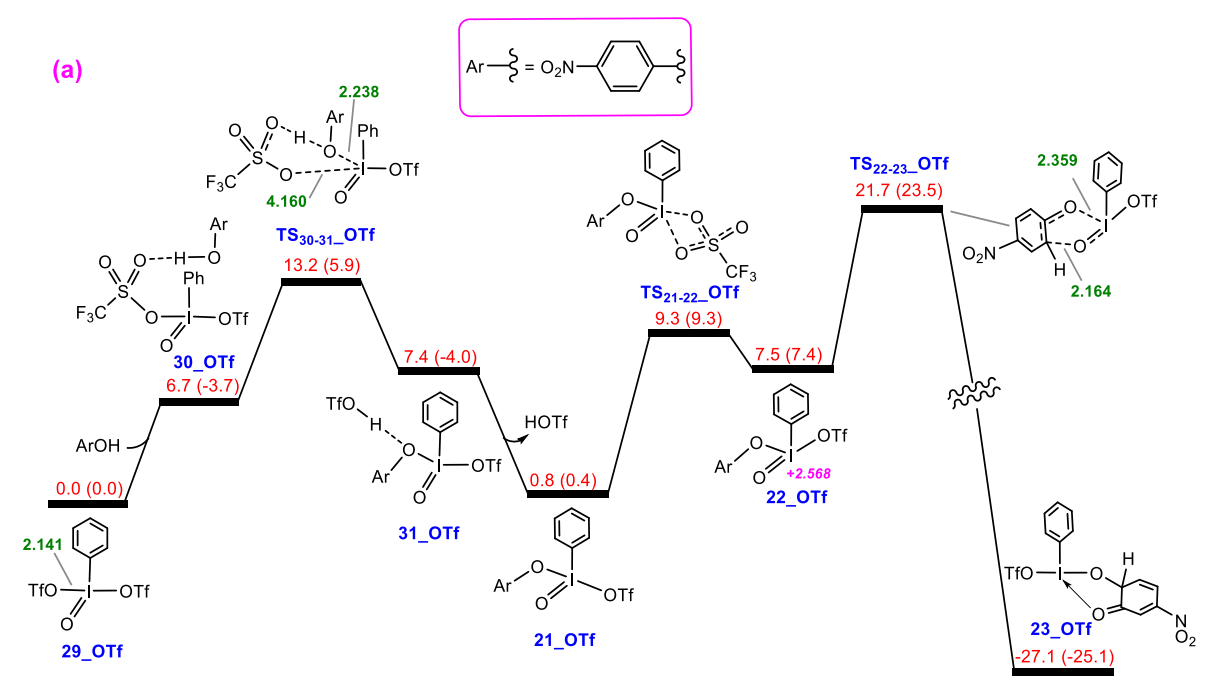

(b)
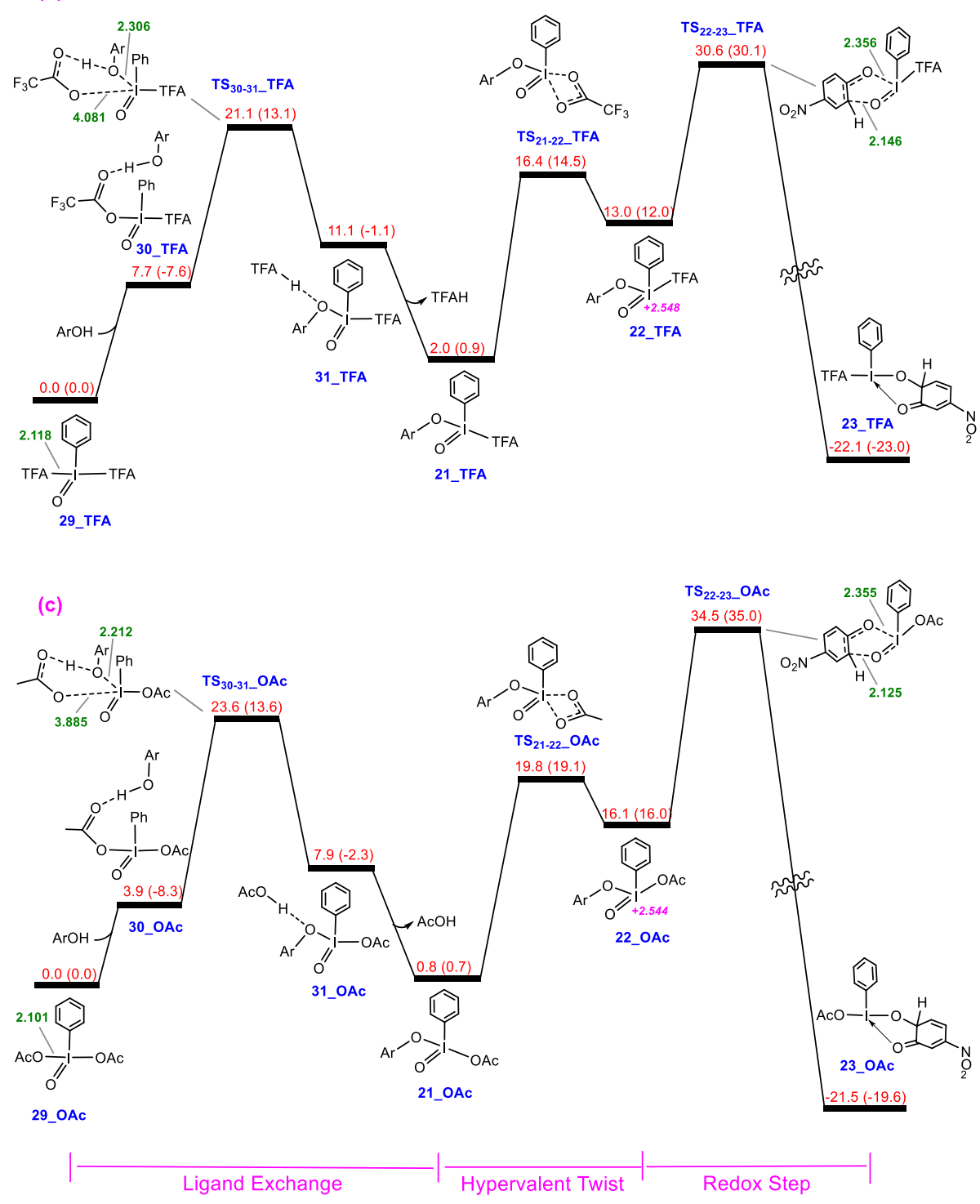

Figure 3. Calculated energy profile for first redox process associated with reaction between 29_X and $\mathrm{ArOH}$ where $\mathrm{X}=\mathrm{OTf}$, TFA, and OAc. Free energies (potential energies) calculated by SMD/M062X/def2-TZVP//SMD/M06-2X/LANL2DZ(d),6-31G(d) in chloroform are given in $\mathrm{kcal} / \mathrm{mol}$ and selected bond distances (green values) in $\AA$. 
Table 2. Reaction free energies for transformations 29_X $\mathbf{X} \mathbf{2 1} \mathbf{X}\left(\Delta \mathrm{G}_{1}\right)$ and $\mathbf{2 1} \mathbf{X} \rightarrow \mathbf{2 2} \_\mathbf{X}\left(\Delta \mathrm{G}_{2}\right)$ and activation free energy for the first redox step (transformation $\mathbf{2 2} \_\mathbf{X} \rightarrow \mathbf{T S}_{22-23} \mathbf{X}, \Delta \mathrm{G}^{\dagger}$ ). Free energies calculated by SMD/M06-2X/def2-TZVP//SMD/M06-2X/LANL2DZ(d),6-31G(d) in chloroform are given in $\mathrm{kcal} / \mathrm{mol}$.

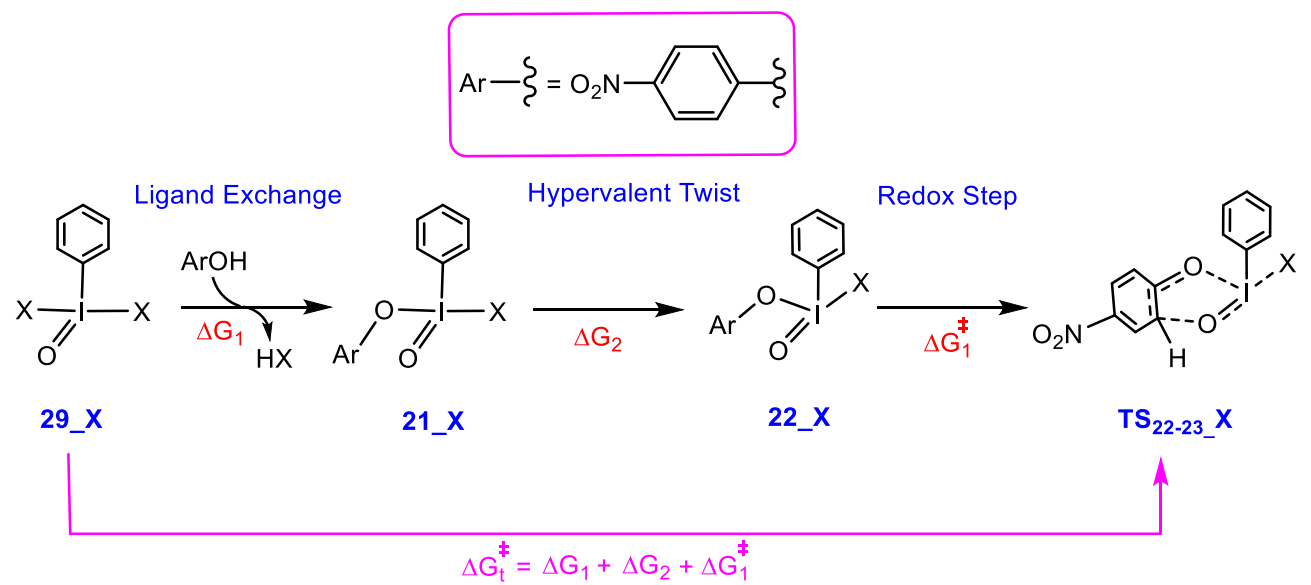

\begin{tabular}{|c|c|c|c|c|}
\hline $\mathbf{X}$ & $\Delta \mathbf{G}_{\mathbf{1}}$ & $\Delta \mathbf{G}_{\mathbf{2}}$ & $\Delta \mathbf{G}^{*} \mathbf{1}$ & $\Delta \mathbf{G}_{\mathbf{t}}^{*}$ \\
\hline OTf & 0.8 & 6.7 & 14.2 & 21.7 \\
\hline TFA & 2.0 & 11.0 & 17.6 & 30.6 \\
\hline OAc & 0.8 & 15.3 & 18.4 & 34.5 \\
\hline
\end{tabular}

Oxidation of $\boldsymbol{p}$-nitrophenol by IBX. We recently investigated the mechanism of the double oxidation of phenols by IBX. ${ }^{10 a}$ Figure 4 depicts the free energy changes for the three key steps outlined in Table 2 . The complete energy profile for the IBX-mediated the oxidation of $p$-nitrophenol is provided in the Supporting Information (Figure S5). Consistent with the experimental findings (Table 1), we found that unlike $\mathrm{PhI}(\mathrm{O})(\mathrm{OTf})_{2}$, the oxidation of $p$-nitrophenol by IBX requires a high overall activation barrier $(30.9 \mathrm{kcal} / \mathrm{mol}) .{ }^{11}$ Since the carboxylate ligand within IBX coordinates more strongly than the triflate, both the $\Delta \mathrm{G}_{2}$ and $\Delta \mathrm{G}^{+}{ }_{1}$ values for IBX are

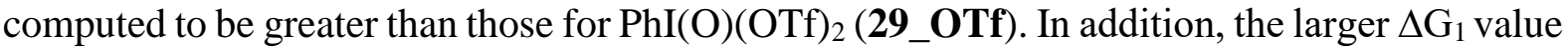
for $\mathrm{IBX}$ than $\mathrm{PhI}(\mathrm{O})(\mathrm{OTf})_{2}$ indicates that the former is thermodynamically more resistant to ligand exchange. It follows from these results that all three key steps (ligand exchange, 
hypervalent twist, and the redox step) contribute to the much lower reactivity of IBX toward oxidation of electron-deficient phenols.

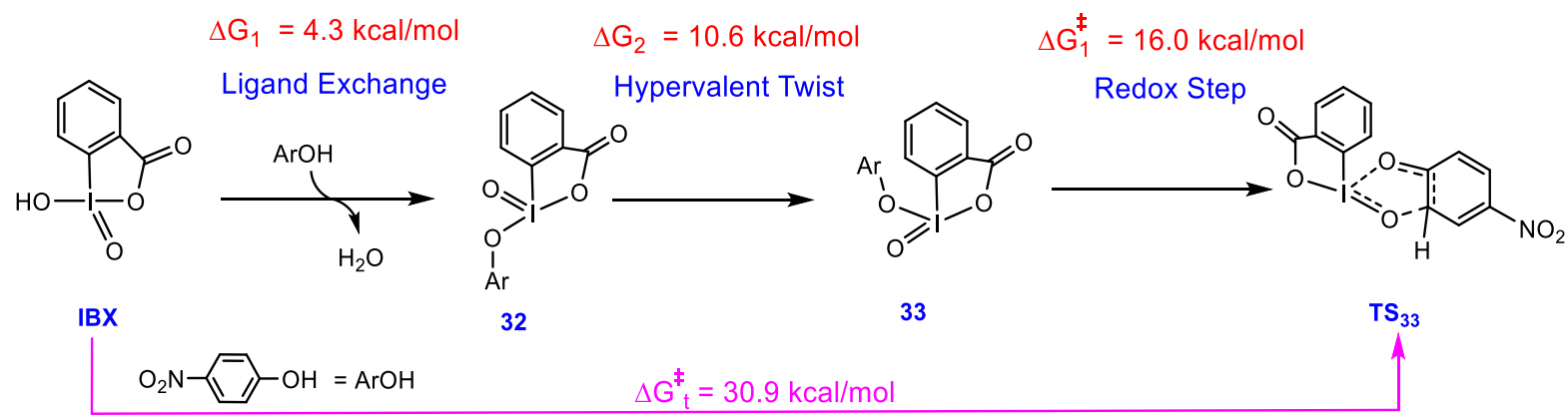

Figure 4. Reaction free energies for the oxidation of $p$-nitro-phenol by IBX. Free energies calculated by SMD/M06-2X/def2-TZVP//SMD/M06-2X/LANL2DZ(d),6-31G(d) in chloroform are given in $\mathrm{kcal} / \mathrm{mol}$.

Oxidation of $p$-nitro-phenol by $\operatorname{PhI}(\mathbf{O})(\mathbf{P y})_{2}(\mathrm{OTf}) 2$. It was determined experimentally that substituting the bidentate bipyridine ligand with two pyridine ligands results in deactivation of the $\operatorname{Bi}(N)$-HVI oxidant and recovery of the starting material (Table 1 , entry 8$).{ }^{5}$ This can be probed by DFT, which predicts that geometrical isomer 36 is $\sim 12 \mathrm{kcal} / \mathrm{mol}$ more stable than the active oxidant 34 (Figure 5). The latter is produced only after surmounting an activation barrier as high as $27.7 \mathrm{kcal} / \mathrm{mol}$. The most stable isomer $\mathbf{3 4}$ is not reactive toward the ligand exchange process because the two active sites in this complex are blocked by the pyridine ligands. Specifically, in the most stable structure, the triflate ligands responsible for driving the ligand exchange occupy positions trans to the strong $\sigma$-donor phenyl and oxo ligands which renders this complex incapable of facilitating the oxidation. This hypothesis is supported by the highly unstable nature of intermediates $\mathbf{3 7}$ and $\mathbf{3 8}$ resulting from ligand exchange between 34 and $\mathrm{ArOH}$. To summarize, the high stability of the inactive iodine(V) complex $\mathbf{3 4}$ does not allow ligand exchange to easily occur on such a system, and this is the likely reason for no reaction occurring between $\mathrm{PhI}(\mathrm{O})(\mathrm{Py})_{2}(\mathrm{OTf})_{2}$ and $p$-nitrophenol. ${ }^{11}$ 


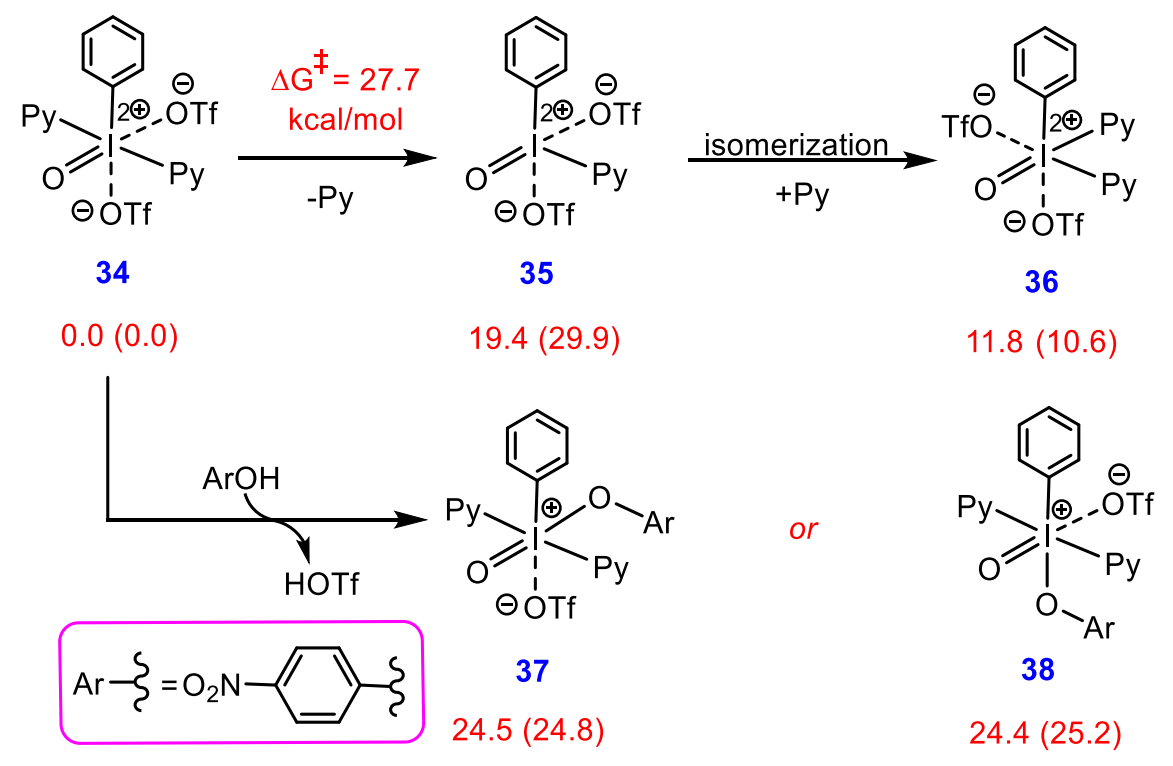

Figure 5. Calculated mechanism for generation of active oxidant $\mathbf{3 6}$ from the most stable isomer $\mathbf{3 4}$ along with the relative energy of intermediates $\mathbf{3 7}$ and $\mathbf{3 8}$ achieved by ligand exchange reaction between 34 and $p$-nitrophenol (ArOH). Free energies (potential energies) calculated by SMD/M06-2X/def2TZVP//SMD/M06-2X/LANL2DZ(d),6-31G(d) in chloroform are given in $\mathrm{kcal} / \mathrm{mol}$.

Oxidation of $p$-nitro-phenol by Bi(4-OMebipy)-HVI. Finally, we wanted to understand why the yield of the oxidative reaction is reduced by replacing the 4,4'-ester substituents within $\mathrm{Bi}\left(4-\mathrm{CO}_{2}\right.$ Etbipy)-HVI with strongly electron-donating 4,4'-dimethoxy moieties (Table 1, entries 1 and 7). ${ }^{5}$ From the DFT-derived mechanism illustrated in Scheme 1, we posit that the reaction between $p$-nitrophenol and $\mathrm{Bi}(4-$ OMebipy)-HVI should commence with the ligand exchange followed by protonation of the bipyridine ligand to give 19_OMe (Figure 6). The overall activation free energy for these two key steps is computed to be only $10.1 \mathrm{kcal} / \mathrm{mol} .{ }^{11}$ Based on the proposed mechanism, for the redox process to proceed, the protonated bipyridine ligand needs to dissociate from 19_OMe to give key intermediate 22_OTf. The presence of the electron-donating 4,4'-dimethoxy groups on the bipyridine ligand cause this key step to take place with an activation barrier of $25.1 \mathrm{kcal} / \mathrm{mol}$, which is much more energy demanding in comparison to analogous process from 19 (18.8 kcal/mol, Figure $2 \mathrm{a})$. The stronger coordination of the bipyridine ligand within 19_OMe than in 19 is evident from the shorter I$\mathrm{N}$ bond distance in the former $(2.336 \AA$ ) than in the latter $(2.426 \AA$ ) (see Figures 6 and $2 \mathrm{a}$ ). 
Consequently, $\mathrm{Bi}\left(4-O M e\right.$ ipy)-HVI is less reactive than $\mathrm{Bi}\left(4-\mathrm{CO}_{2}\right.$ Etbipy)-HVI toward the phenol oxidation, likely because the more tightly coordinated 4,4'-methoxy bipyridine ligand retards the formation of key intermediate 22_OTf.

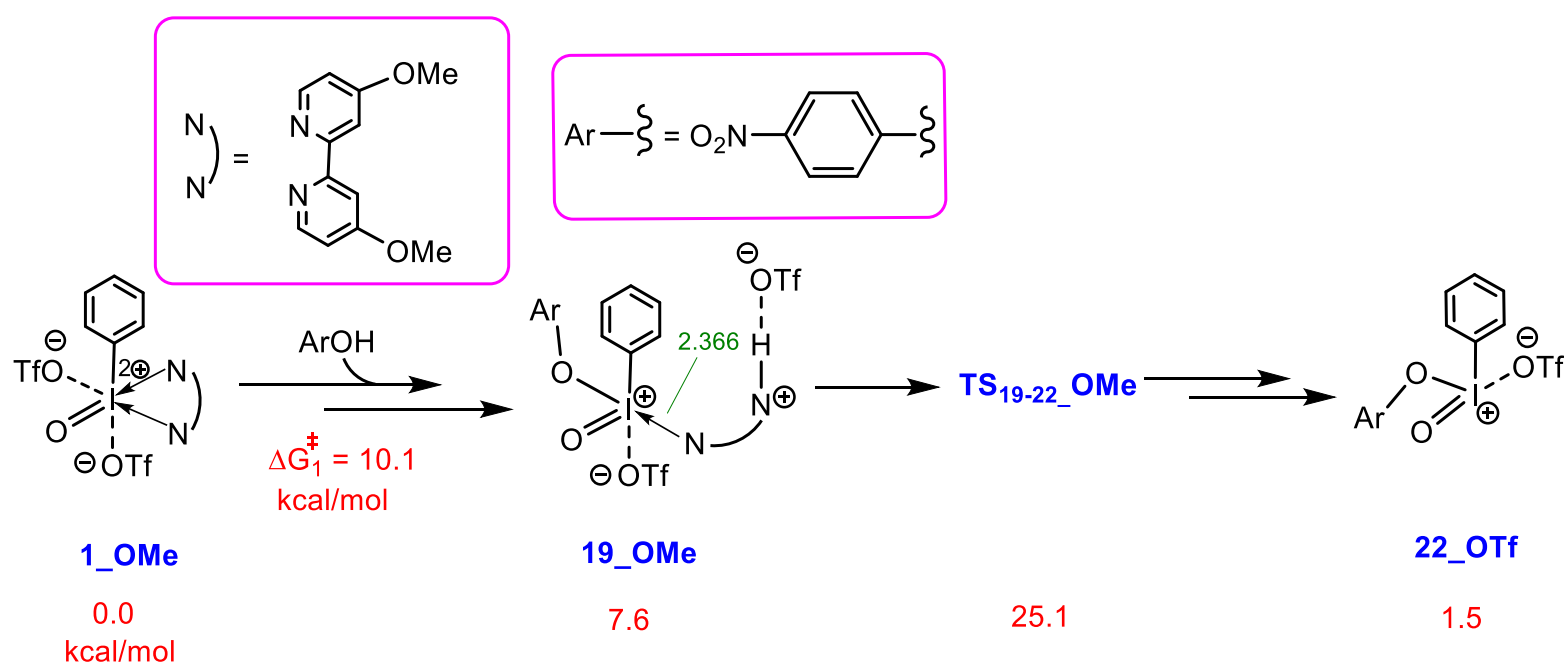

Figure 6. Calculated mechanism for formation of the key intermediate 22 OTf from reaction between 1_OMe and $p$-nitrophenol (ArOH). Free energies calculated by SMD/M06-2X/def2TZVP//SMD/M06-2X/LANL2DZ(d),6-31G(d) in chloroform are given in $\mathrm{kcal} / \mathrm{mol}$.

\section{CONCLUSION}

In summary, our comprehensive computational investigation reveals how the key structural and electronic properties of $\mathrm{Bi}\left(4-\mathrm{CO}_{2}\right.$ Etbipy)-HVI facilitate its enhanced oxidation capacity relative to more traditional hypervalent iodine $(\mathrm{V})$ species. We anticipate that these findings will assist in the design and development of new hypervalent iodine(V) reagents and synthetic transformations.

\section{COMPUTATIONAL METHODS}

Gaussian $16^{12}$ was used to fully optimize all the structures reported in this paper at the M06$2 \mathrm{X}$ level ${ }^{13}$ of density functional theory (DFT). For all the calculations, solvent effects were considered using the SMD solvation model for $\mathrm{CHCl}_{3}$ solvent. ${ }^{14}$ The effective core potential of 
Hay and Wadt with a double- $\xi$ valence basis set (LANL2DZ) ${ }^{15}$ was chosen to describe iodine. Polarization functions were also added for $\mathrm{I}(\xi \mathrm{d}=0.289) .{ }^{16}$ The $6-31 \mathrm{G}(\mathrm{d})$ basis set was used for other atoms. ${ }^{17}$ This basis set combination will be referred to as BS1. Frequency calculations were carried out at the same level of theory as those for the structural optimization. Transition structures were located using the Berny algorithm. Intrinsic reaction coordinate (IRC) calculations ${ }^{18}$ were used to confirm the connectivity between transition structures and minima. To further refine the energies obtained from the SMD/M06-2X /BS1 calculations, we carried out single-point energy calculations using the M06-2X functional method ${ }^{19}$ for all the structures with a larger basis set (BS2). BS2 utilizes def2-TZVP ${ }^{20}$ basis set on all atoms. Tight convergence criterion was also employed to increase the accuracy of the single point calculations. All thermodynamic data were calculated at the standard state $(298.15 \mathrm{~K}$ and 1 atm).

In this work, the free energy for each species optimized by SMD/M06-2X/BS1 in solution was calculated using the formula: $\mathrm{G}=\mathrm{E}(\mathrm{BS} 2)+\mathrm{G}(\mathrm{BS} 1)-\mathrm{E}(\mathrm{BS} 1)+\Delta \mathrm{G}^{1 \mathrm{~atm} \rightarrow 1 \mathrm{M}}$; where $\Delta \mathrm{G}^{1 \mathrm{~atm} \rightarrow 1 \mathrm{M}}$ $=1.89 \mathrm{kcal} / \mathrm{mol}$ is the free-energy change for compression of 1 mole of an ideal gas from $1 \mathrm{~atm}$ to the $1 \mathrm{M}$ solution phase standard state. Natural population analysis (NPA) was carried out using NBO6 software integrated into Gaussian $16 .^{21}$

The free energy barriers for ligand dissociation via TS19-20, TS34-35, TS18-20 and TS19-22-OMe were estimated according to the protocol presented by Hall and Hartwig. ${ }^{22}$ In this protocol, for example, the Gibbs free energy barrier for a dissociation reaction such as $\mathrm{A}-\mathrm{B} \rightarrow \mathrm{A}+\mathrm{B}$ is estimated as $\Delta \mathrm{G}^{\ddagger} \approx \Delta \mathrm{H}=\mathrm{H}_{\mathrm{A}}+\mathrm{H}_{\mathrm{B}}-\mathrm{H}_{\mathrm{A}-\mathrm{B}}$.

\section{ASSOCIATED CONTENT}

\section{Supporting Information}

The Supporting Information is available free of charge on the ACS Publications website. 


\title{
AUTHOR INFORMATION
}

\section{Corresponding Authors}

\author{
*E-mail: Alireza.Ariafard@utas.edu.au. \\ orcid.org/0000-0003-2383-6380 \\ *E-mail: SarahW@temple.edu. \\ orcid.org/0000-0002-4797-0181
}

\section{Notes}

The authors declare no competing financial interest.

\section{ACKNOWLEDGMENTS}

We thank the Australian Research Council (ARC) for project funding (DP180100904) and the Australian National Computational Infrastructure and the University of Tasmania for the generous allocation of computing time. A.C.B.'s contributions were supported by an ARC Future Fellowship (FT200100049).

\section{REFERENCES}

(1) (a) Zhandkin, V. V.; Stang, P. J. Chemistry of Polyvalent Iodine. Chem. Rev. 2008, 108, 5299-5358. (b) Charpentier, J.; Fruh, N.; Togni, N. A. electrophilic Trifluoromethylation by Use of hypervalent Iodine Reagents. Chem. Rev. 2015, 115, 650-682. (c) Yoshimura, A.; Yusubov, M.; Zhdankin, V. V. Synthetic applications of Pseudocyclic Hypervalent Iodine compounds. Org. Biomol. Chem. 2016, 14, 4771-4781. (d) Kohlhepp, S. V.; gulder, T. Hypervalent Iodine (III) Fluorinations of Alkenes and Diazo compounds: New Opportunities in fluorination chemistry. Chem. Soc. Rev. 2016, 45, 6270-6288.(e) Yoshimura, A.; Zhdankin, V. V. Advances in Synthetic Applications of Hypervalent Iodine compounds. Chem. Rev. 2016, 116, 33283435 .

(2) (a) Ladziata, U.; Zhandkin, V. V. Hypervalent Iodine (V) Reagents in Organic synthesis. Arkivoc. 2006, ix, 26-58. (b) Satam. V.; Harad, A.; Rajule, R.; Pati, H. 2-Iodoxybenzoic acid (IBX): an Eficient Hypervalent Iodine Reagent. Tetrahedron. 2010, 66, 7659-706. (c) Zhandkin, V. V. Organoiodine (V) Reagents in Organic Synthesis. J. Org. Chem. 2011, 76, 1185-1197. (d) Duschek, A.; Kirsch, S. F. 2-Iodoxybenzoic acid-A Simple Oxidant with a Dazzling Array of Potential Applications. Angew. Chem. Int. Ed. 2011, 50, 1524-1552. (e ) Bernini, R.; Fabrizi, G.; Pouysegu, L.; Defiieux, D.; Quideau, S. Synthesis of biologically Active Catecholic Compounds via ortho-Selective Oxygenation of Phenolic Compounds Using Hypervalent Iodine (V) Reagents. Curr. Org. Synth. 2012, 9, 650-669.

(3) For the first example of a regioselective ortho-quinone synthesis using IBX, see: a) Magdziak, M.; rodriguez, R. W.; De Water, R. W.; Petus, T. R. R. Org. Let. 2002, 4, 285-28. b) Tohma, h.; Kita, Y. Hypervalent Iodine Reagents for Oxidation of alcohols and their application to complex molecule synthesis. Adv. Synth. Catal. 2004, 346, 111. (c) Uyanik, M.; Mutsuga, T.; Ishihara, K. Angew. Chem. Int. Ed. 2017, 56, 3956-3960. (d) Stack. D. E.; Mahmud, B. Synth. Commun. 2018, 48, 161-167.

(4) Zhdankin, V. V.; Koposov, A. Y.; Yashin, N. V. complexes of Hypervalent Iodine Compounds with nitrogen Ligands. Tetrahedron Lett. 2002, 43, 5735-5737.

(5) Xiao, X.; Greenwood, N. S.; Wengriniuk, S. E. Dearomatization of electron-deficient Phenols to orthoQuinones: Bidentate Nitrogen-Ligated Iodine(V) Reagents. Angew. Chem. Int. Ed. 2019, 58, 16181-16187. 
(6) Xiao, X.; Roth, J. M.; Greenwood, N. S.; Veloplcek, M. K.; Aquirre, J.; Jalali, M.; Ariafard, A.; Wengryniuk, S. E. Bidentate Nitrogen-Ligated I(V) Reagents, Bi(N)-HVIs: Preparation, stability, structure, and Reactivity. J. Org. Chem. 2021, 86, 6566-6576.

(7) (a) Ganji, B.; Ariafard, A. DFT Mechanistic Investigation into Phenol Dearomatization Mediated by an Iodine (III) Reagent. Org. Biomol. Chem. 2019, 17, 3521-3528. (b) Farshadfar, K.; Chipman, A.; Yates, B. F.; Ariafard, A. DFT Mechanistic Investigation into BF3-catalyzed Alcohol Oxidation by a hypervalent Iodine (III) Compound. ACS. Catal. 2019, 9, 6510-6521.

(8) The dissociation of the bipyridine ligand from 18 gives 20 with a relative energy of $26.1 \mathrm{kcal} / \mathrm{mol}$ and occurs with an overall activation barrier of $\sim 31.6 \mathrm{kcal} / \mathrm{mol}$, indicating that the protonation of the $\mathrm{N}^{\mathrm{b}}$ atom by triflic acid is particularly necessary for the bipyridine ligand to be liberated more easily.

(9) (a) Su, J. T.; Goddard, W. A. Enhancing 2-Iodoxybenzoic Acid Reactivity by Exploiting a Hypervalent Twist. J. Am. Chem. Soc. 2005, 127, 14146-14147. (b) jiang, H.; Sun, T. Y.; Wang, X.; Xie, Y.; Zhang, X.; Wu, Y. D.; Schaefer, H. F. A Twist of the Twist Mechanism, 2-Iodoxybenzoic Acid (IBX)-Mediated Oxidation of Alcohol Revisited: theory and Experiment. Org. Lett. 2017, 19, 6502-6505.

(10) Kaur, A.; Ariafard, A. Mechanistic Investigation into Phenol Oxidation by IBX Elucidated by DFT Calculations. Org. Biomol. Chem. 2020, 18, 1117-1129.

(11) For the full energy profile, see the Supporting Information.

(12) Gaussian 16, Revision B.01, Frisch, M. J.; Trucks, G. W.; Schlegel, H. B.; Scuseria, G. E.; Robb, M. A.; Cheeseman, J. R.; Scalmani, G.; Barone, V.; Petersson, G. A.; Nakatsuji, H.; Li, X.; Caricato, M.; Marenich, A. V.; Bloino, J.; Janesko, B. G.; Gomperts, R.; Mennucci, B.; Hratchian, H. P.; Ortiz, J. V.; Izmaylov, A. F.; Sonnenberg, J. L.; Williams-Young, D.; Ding, F.; Lipparini, F.; Egidi, F.; Goings, J.; Peng, B.; Petrone, A.; Henderson, T.; Ranasinghe, D.; Zakrzewski, V. G.; Gao, J.; Rega, N.; Zheng, G.; Liang, W.; Hada, M.; Ehara, M.; Toyota, K.; Fukuda, R.; Hasegawa, J.; Ishida, M.; Nakajima, T.; Honda, Y.; Kitao, O.; Nakai, H.; Vreven, T.; Throssell, K.; Montgomery, J. A.; Peralta, J. E.; Ogliaro, F.; Bearpark, M. J.; Heyd, J. J.; Brothers, E. N.; Kudin, K. N.; Staroverov, V. N.; Keith, T. A.; Kobayashi, R.; Normand, J.; Raghavachari, K.; Rendell, A. P.; Burant, J. C.; Iyengar, S. S.; Tomasi, J.; Cossi, M.; Millam, J. M.; Klene, M.; Adamo, C.; Cammi, R.; Ochterski, J. W.; Martin, R. L.; Morokuma, K.; Farkas, O.; Foresman, J. B.; and Fox, D. J. Gaussian, Inc., Wallingford CT, 2016.

(13) (a) Lee, C. T.; Yang, W. T.; Parr, R. G. Development of the Colic-Salvetti Correlation-energy Formula into a Functional of the Electron Density. Phys. Rev. B. 1988, 37, 785-789; (b) Miehlich, B.; Savin, A.; Stoll, H.; Preuss, H. Results Obtained with the Correlation Energy Density Functionals of Becke and Lee, Yang and Parr. Chem. Phys. Lett. 1989, 157, 200-206; (c) Becke, A. D. J. Density Functional Thermochemistry.III. The Role of Exact Exchange. Chem. Phys. 1993, 98, 5648-5652.

(14) Marenich, A. V.; Cramer, C. J.; Truhlar, D. G. Universal Solvation Model Based on Solute Electron Density and on a continuum Model of the Solvent defined by the Bulk Dielectric Constant and Atomic Surface Tensions. J. Phys. Chem. B. 2009, 113, 6378-6396.

(15) (a) Hay, P. J.; Wadt, W. R. Ab initio Effective Core Potentials for Molecular Calculations. Potentials for the Transition Metal Atoms Sc to Hg. J. Chem. Phys. 1985, 82, 270-283. (b) Wadt, W. R.; Hay, P. J. Ab initio Effective Core Potentials for Molecular Calculations. Potentials for Main Group elements Na to Bi. J. Chem. Phys. 1985, 82, 284-298.

(16) Ehlers, A. W.; Dapprich, M. S.; Gobbi, A.; Höllwarth, A.; Jonas, V.; Köhler, K. F.; Stegmann, R.; Veldkamp, A.; Frenking, G. A Set of f-polarization Functions for Pseudo-potential Basis Sets of the Transition Metals Sc, Cu, Y, Ag, La, Au. Chem. Phys. Lett. 1993, 208, 111-114.

(17) Hariharan, P. C.; People, J. A. The Influence of Polarization Functions on Molecular Orbital Hydrogenation Energies. Theor. Chim. Acta J. A. 1973, 28, 213-222.

(18) (a) Fukui, K. J. Formulation of the Reaction coordinate. Phys. Chem. 1970, 74, 4161-4163; (b) Fukui, K. The Path of The Chemical Reactions -The IRC Approach. Acc. Chem. Res. 1981, 14, 363-368.

(19)Zhao, Y.; Truhlar, D. G. Density Functionals with Broad Applicability in Chemistry. Acc. Chem. Res. 2008, 41, 157-167.

(20) Weigend, F.; Furche, F. and Ahlrichs, R. J. Gaussian Basis Sets of Quadruple Zeta Valence Quality for Atoms H-Kr. Chem. Phys. 2003, 119, 12753-12762.

(21) Glendening, E. D.; Badenhoop, J. K.; Reed, a. E.; Carpenter j. E.; Bohmann, J. A.; Morales, C. M.; Landis, C. R.; Weinhold, F. Natural Bond Order 6.0, Theoretical Chemistry Institute, University of Wisconsin, Madison, WI, 2013, http://nbo6.chem.wisc.edu.

(22) Hartwig, J. F.; Cook, K. S.; Hapke, M.; Incarvito, C. D.; Fan, Y.; Webster, C. E.; Hall, M. B. Rhodium Boryl Complexes in the Catalytic, Terminal Functionalization of Alkanes. J. Am. Chem. Soc. 2005, 127, 2538-2552. 